\title{
Superhard materials: recent research progress and prospects
}

\author{
Bo $\mathrm{Xu}$ and Yongjun Tian*
}

\begin{abstract}
Over the past several decades, great endeavors have been devoted to superhard materials research, among which two topics are of central focus. One is to understand hardness microscopically and reveal the controlling factors for superhardness, which can be used to guide the design of novel superhard crystals; the other is to synthesize superhard materials with enhanced comprehensive performance (i.e., hardness, fracture toughness, and thermal stability), with the ambition to synthesize materials harder than natural diamond. We proposed a microscopic understanding of the indentation hardness as the combined resistance of chemical bonds in a material to indentation, and established a microscopic hardness model for covalent and polar covalent crystals, which was further generalized to polycrystalline materials. Guided by the polycrystalline hardness model, we successfully synthesized nanotwinned cubic boron nitride and diamond bulks under high pressure and high temperature. These materials exhibit simultaneous improvement of hardness, fracture toughness, and thermal stability, designating a new direction for superhard materials research.
\end{abstract}

\section{INTRODUCTION}

Superhard materials are defined as materials with Vickers hardness higher than $40 \mathrm{GPa}$ [1]. This class of materials display superior mechanical performance in hardness, incompressibility, toughness, and wear resistance, which can be utilized in a wide range of applications, such as cutting and polishing tools in machinery industry, drilling bits in milling and petrochemical industry, and diamond anvil cells in high pressure science. Diamond and cubic boron nitride $(\mathrm{cBN})$, both of which can be produced massively [2,3], are the best-known superhard materials. Unfortunately, each of them shows some inherent shortcomings, such as the inferior thermal stability for diamond, as well as the relatively low hardness and fracture toughness for $\mathrm{cBN}$. Syntheses of superhard materials with improved comprehensive performance, i.e., simultaneously improved hardness, toughness, and thermal stability, are thus highly desired [4-9]. In addition, synthesizing materials harder than natural diamond has always been a sought-after goal of human beings [10]. In order to achieve these goals, a fundamental understanding of the physical origin of materials hardness is necessary.
Superhard materials are characterized by hardness. Macroscopically, hardness can be defined as the ability of a material to resist being scratched or dented by another. By such a definition, hardness has to be classified as an engineering quantity, and distinct hardness scales have been developed experimentally depending on the specific measurement methods (scratch, indentation, or rebound). This situation causes some difficulties in hardness prediction. For example, Mohs hardness measures the ability of one material to scratch another and is quantified by an integer (or half-integer) from 0 to 10 on the Mohs scale, which obviously is discrete and non-linear. At present, indentation hardness scales (especially the Vickers and Knoop scales differing from each other in the shape of diamond indenter) are commonly used for hardness determination. Indentation hardness measures a material's resistance to permanent plastic deformation resulting from a compressive load by a sharp indenter, and can be calculated from the indenter load divided by the contact (or projected) area of the permanent indentation formed on the sample surface [11]. Vickers hardness, for example, is calculated as $H_{\mathrm{V}}(\mathrm{GPa})=$ $1854.4 \mathrm{~L} / \mathrm{d}^{2}$, where load $(L)$ and average diagonal length of indentation $(d)$ are in the units of $\mathrm{N}$ and $\mu \mathrm{m}$, respectively. Usually, the calculated hardness depends on the shape of the indenter, loading force and rate, indentation size and time, sample orientation, as well as surface condition. With all these factors taken into account, an essentially aptotic hardness value can be assigned to a specific material from the asymptotic-hardness region of a well-controlled indentation process, providing a solid experimental basis to investigate the fundamental factors controlling materials hardness.

Hardness prediction has been a great challenge for materials scientists. Traditionally, empirical models correlate hardness with the elastic properties of crystals [12-14]. However, such correlations are physically questionable since hardness characterizes a permanent plastic deformation, rather than a reversible elastic deformation [15]. In 2003, Tian's group [16] intuitively but constructively proposed a microscopic picture of hardness: it can be defined

State Key Laboratory of Metastable Materials Science and Technology, Yanshan University, Qinhuangdao 066004, China

* Corresponding author (email: fhcl@ysu.edu.cn) 
as the combined resistance of chemical bonds in a material to indentation. Based on this understanding and available experimental data, they established a microscopic hardness model to evaluate the intrinsic hardness of covalent and polar covalent crystals $[16,17]$, which was later generalized to including the effects of metallicity and polycrystallinity $[18,19]$. Following this success, other hardness models with similar considerations were proposed [20,21]. In these models, microscopic parameters (e.g., bond length, valence electron density, ionicity) that can easily be determined for a specific crystal are used to estimate materials hardness. Combined with state-of-the-art crystal structure prediction methods, these models provide a powerful tool for theoretical design of superhard materials.

Along with these theoretical advances of hardness calculations, experimental progresses have recently also been achieved in superhard materials research, which can be categorized into two strategies. One is to design and synthesize new superhard materials, and two classes of materials are of special interest. The first class includes light-element compounds made of boron, carbon, nitrogen, and oxygen. In these systems, the intrinsically strong and directional covalent bonds of the light elements lead to tight three dimensional bonding networks with extreme resistance to external shear, which are essential to realize superhardness. Significant progresses have been achieved in synthesizing superhard $\mathrm{BC}_{x} \mathrm{~N}, \mathrm{BC}_{x}, \gamma \mathrm{B}_{28}, \mathrm{~B}_{6} \mathrm{O}$ [22-27]. The second class consists of materials made of transition metals and light elements (B, C, and $\mathrm{N}$ ), such as $\mathrm{ReB}_{2}, \mathrm{OsB}_{2}, \mathrm{WB}_{4}, \mathrm{FeB}_{4}, \mathrm{PtC}$, $\mathrm{IrN}_{2}, \mathrm{OsN}_{2}$, and $\mathrm{PtN}_{2}$ [28-36]. It was proposed that transition metals could introduce a high valence electron density to resist the elastic deformations while lights elements can introduce short and strong covalent bonds to prevent atoms from slipping under stress. Although the finding of superhard materials in this class of materials is not successful yet [34,37-39], it provides a great searching pool for potential superhard materials. The other strategy is to enhance hardness of known materials through appropriate design or control of their microstructure. Following this strategy, nanocrystalline $\mathrm{TiN} / \mathrm{SiN}_{x}$ nanocomposites were synthesized with hardness exceeding $100 \mathrm{GPa}$ [9]. This hardness value is remarkably high especially when one considers the fact that both components, $\mathrm{TiN}$ and $\mathrm{SiN}_{x}$, are just hard materials. The hardness of diamond and $\mathrm{cBN}$ was also enhanced through formation of nanocrystalline microstructures [40-42]. Most recently, we synthesized nanotwinned $\mathrm{cBN}$ (nt-cBN) and nanotwinned diamond (nt-diamond) with marvelous hardness enhancement as well as simultaneous improvement in fracture toughness and thermal stability $[43,44]$.

In this review, recent progresses in superhard materials are presented. We start with the microscopic hardness model for covalent and polar covalent crystals as well as its generation to polycrystalline materials. Following that, our recent works on ultrahard nt-cBN and nt-diamond are discussed. A long-standing controversy about the criterion to perform a reliable indentation hardness measurement is clarified in the next section. We conclude this review with discussions of future developments in superhard materials. We have to point out that this review is not intended to be exhaustive, and is mainly focused on our own work with necessary discussion on selected important research from other groups. Several excellent reviews provided a more comprehensive overview on superhard materials research $[1,4-7]$, which interested audience can refer to.

\section{UNDERSTANDING OF HARDNESS}

Historically, researchers suggested linear correlations between indentation hardness and bulk modulus $(B)$ or shear modulus $(G)$ [12-14]. However, it is now accepted that hardness does not depend linearly on $B$ or $G$ because hardness characterizes a permanent plastic deformation, while elastic moduli correspond to reversible elastic deformations [15]. Chen et al. [15] later proposed a macroscopic model based on $G$ and Pugh's modulus ratio $(k=G / B)$. The value of $k$ is closely correlated to the brittleness/ductility of materials, and highlights the relationship between elastic and plastic properties: brittle materials have high ratios while ductile materials have low ones [45]. In principle, covalent materials with high hardness are obviously brittle with a large $k$. In this macroscopic model, the Vickers hardness of polycrystalline materials can be estimated according to $H_{\mathrm{V}}$ $=2\left(k^{2} G\right)^{0.585}-3$, which provides better reliability for hardness evaluation since $k$ responds to both the elasticity and plasticity of materials [15]. Although the origin of hardness at the microscopic level may need to be further elucidated, Chen's macroscopic model provides a very simple method to estimate the hardness of complex crystals, such as porous T-carbon [46]. However, direct quantification of hardness with microscopic parameters (i.e., a microscopic hardness model) would reveal the fundamental factors controlling hardness and provide valuable guidelines for design of new superhard materials.

Hardness quantifies the resistance of a crystal against plastic deformation. The resistance to deformation depends not only on the dislocation density created by a rigid indentation, but also on the original stored dislocation density [47]. The stored dislocation density in metals is usually high, leading to an extrinsic hardness for metals. We thus limit our discussion to covalent and polar covalent materials with localized chemical bonds. In such materials, the stored dislocation density is negligible. First, let us consider single crystals, for which hardness is intrinsic and entirely depends on the resistance of the chemical bonds in 
the crystal within the indentation area.

When an indenter is pressed into the surface of a single crystal, as shown in Fig. 1a, the chemical bonds in the vicinity of the indenter tip withstand bend, stretch, or compress. Based on this simple physical picture, Tian's group [16] proposed an intuitive and constructive assumption that, for covalent and polar covalent crystals, hardness is equivalent to the sum of the resistance of each bond to the indenter per unit area. The plastic deformation associated with the formation and motion of dislocations is thus related to the breaking of electron-pair bonds in the crystals. Hardness then measures the combined resistance of chemical bonds to indentation: higher density and stronger resistance of chemical bonds would result in harder crystals. In covalent crystals, breaking a chemical bond energetically means that two bonding electrons are excited from the valence band to the conduction band, as shown in Fig. 1b. The activation energy required for a plastic glide is thus twice the band gap $E_{\mathrm{g}}$ [48]. The resistance force of a bond can be evaluated with the corresponding $E_{\mathrm{g}}$. As a result, the hardness of pure covalent crystals can be evaluated as:

$$
H(\mathrm{GPa})=A N_{\mathrm{a}} E_{\mathrm{g}},
$$

where $A$ is a proportional constant, and $N_{\mathrm{a}}$ is the number of covalent bonds per unit area, which is calculated as:

$$
N_{\mathrm{a}}=\left(\sum_{i} n_{i} Z_{i} / 2 V\right)^{2 / 3}=\left(N_{\mathrm{e}} / 2\right)^{2 / 3} .
$$

$n_{i}$ is the number of the $i^{\text {th }}$ atom in the unit cell, $Z_{i}$ is the valence electron number of the $i^{\text {th }}$ atom contributing to bonding, $V$ is the volume of the unit cell, and $N_{\mathrm{e}}$ is the valence electron density.

For polar covalent crystals, the valence electrons are preferentially distributed to the anion side (Fig. 1c), which weakens the binding of two atoms. This adverse contribution from the bond ionicity needs to be considered in the hardness evaluation of polar covalent crystals. Phillips [49] suggested that $E_{\mathrm{g}}$ for a binary polar covalent crystal can be separated into a covalent homopolar component, $E_{\mathrm{h}}$, and an ionic heteropolar one, $C$, as $E_{\mathrm{g}}^{2}=E_{\mathrm{h}}^{2}+C^{2}$. $E_{\mathrm{h}}$ determines the activation energy of a dislocation glide in polar covalent crystals [50], and can be estimated in electronvolt with the empirical expression $E_{\mathrm{h}}=39.74 d^{-2.5}$, where $d$ is the bond length in angstroms [49]. The ionic contribution results in a loss of covalent bond charge and is accounted for by introducing a correction factor, $\mathrm{e}^{-\alpha f_{i}}$, to Equation (1). This correction factor describes the screening effect for each bond, where $\alpha$ is a constant and $f_{\mathrm{i}}=1-E_{\mathrm{h}}^{2} / E_{\mathrm{g}}^{2}$ is the Phillips ionicity of the chemical bond in a crystal [49]. The constants $A$ and $\alpha$, determined by fitting the hardness expression $H_{\mathrm{V}}=A N_{\mathrm{a}} \cdot 39.74 d^{-2.5} \cdot \mathrm{e}^{-\alpha f_{\mathrm{i}}}$ to a experimentally determined Vickers hardness dataset for covalent and polar covalent crystals, are 14 and 1.191, respectively [16]. We thus established a microscopic Vickers hardness model for binary (polar) covalent crystals in terms of $d, f_{\mathrm{i}}$, and $N_{\mathrm{e}}$ as:

$$
H_{\mathrm{V}}(\mathrm{GPa})=556 N_{\mathrm{a}} d^{-2.5} \mathrm{e}^{-1.191 f_{\mathrm{i}}}=350 N_{\mathrm{e}}^{2 / 3} d^{-2.5} \mathrm{e}^{-1.191 f_{\mathrm{i}}} .
$$

This hardness model can easily be applied to a multicomponent system by assuming the average hardness to be the geometrical mean of the hardness of different types of covalent bonds in the system via:

$$
H_{\mathrm{V}}=\left[\prod^{\mu}\left(H_{\mathrm{V}}^{\mu}\right)^{n^{\mu}}\right]^{1 / \sum n^{\mu}},
$$

where $H_{\mathrm{V}}^{\mu}=350\left(N_{\mathrm{e}}^{\mu}\right)^{2 / 3}\left(d^{\mu}\right)^{-2.5} \mathrm{e}^{-1.191 f_{\mathrm{i}}^{\mu}}$ is the hardness of a binary compound composed of $\mu$ bonds, and $n^{\mu}$ is the number of $\mu$-type bonds in the unit cell. Readers can refer to the original paper for further details [16].

The microscopic hardness model presented in Equation (3) was further generalized $[17,18]$, which are presented briefly here. First, a new ionicity scale was proposed based on first-principles calculations [17]. For a specific crystal structure or cluster containing the same type of coordinate configuration, an ionicity scale, $f_{\mathrm{h}}$, can be defined for
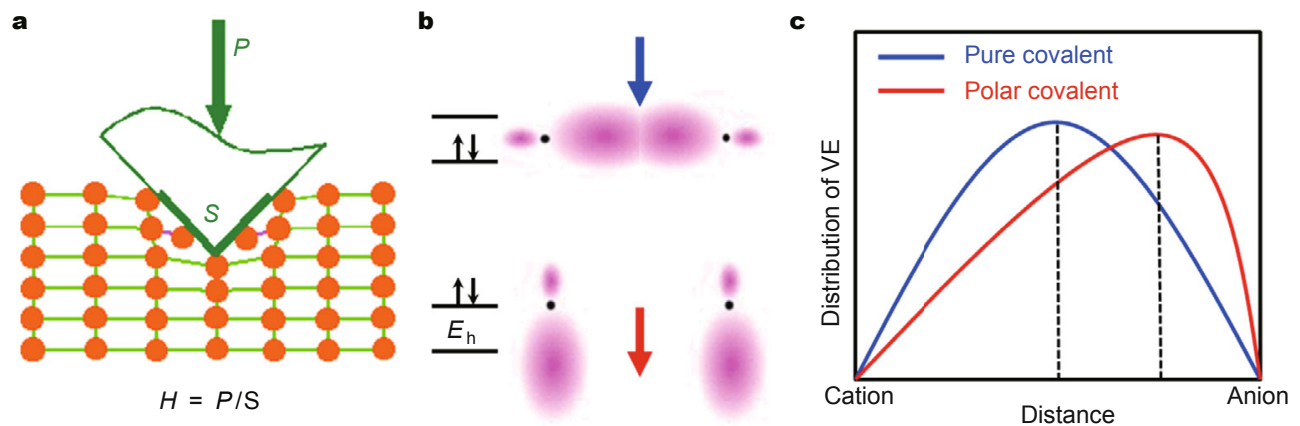

Figure 1 (a) Response of chemical bonds under an indentor during a hardness measurement. (b) Electron excitation accompanying chemical bond breaking. Two bonding electrons are excited from the valence band to the conduction band. (c) The distribution of valence electrons (VE) in the bonding region for pure and polar covalent bonds. Reprinted with permission from Ref. [19]. Copyright 2012, Elsevier. 
a bond based on the Mulliken bond overlap population as $f_{\mathrm{h}}=1-\mathrm{e}^{-\left|P_{\mathrm{c}}-P\right| / P}$, where $P$ is the overlap population of a bond in the calculated crystal, and $P_{c}$ is the overlap population of a bond in a pure covalent crystal with an identical structure to the calculated one. Phillips ionicity $f_{\mathrm{i}}$ can then be replaced by a power law function of $f_{\mathrm{h}}, f_{\mathrm{i}}=f_{\mathrm{h}}^{m}$, where $m=$ 0.735. All the inputs for this hardness model can now be obtained through first-principles calculations.

Later, a factor of metallicity, $f_{\mathrm{m}}=n_{\mathrm{m}} / n_{\mathrm{e}}$, was introduced to account for the effect of metallicity on hardness, where $n_{\mathrm{m}}=k_{\mathrm{B}} T D_{\mathrm{F}}$ is the number of electrons that can be excited at ambient temperature, and $n_{\mathrm{e}}$ is the total number of valence electrons in the unit cell [18]. At ambient temperature, $k_{\mathrm{B}} T$ $\approx 0.026 \mathrm{eV}$ and $D_{\mathrm{F}}$ is the electronic density of states at the Fermi level, which can be acquired via electronic structure calculations. Similar to the contribution of ionicity to hardness [16], the screening effect of the metallic component can be described with a correction factor of $\mathrm{e}^{-\beta f_{\mathrm{m}}^{n}}$, where $\beta$ and $n$ are constants. The contributions from $d$ valence electrons in transition metal compounds to hardness were also considered. The bond strength of an $s-p-d$ hybridized chemical bond is greater than that of an $s$ - $p$ hybridized chemical bond [51]. The intrinsic influence of the $d$ valence electrons should result in a proportional coefficient $A^{\prime}$ for $s$ - $p$ - $d$ hybridized crystals different from that for the $s-p$ hybridized crystals in Equation (1). Including contributions from both metallicity and $s-p$ - $d$ hybridization, the hardness equation is updated to [18]:

$$
H_{\mathrm{V}}(\mathrm{GPa})=1051 N_{\mathrm{e}}^{2 / 3} d^{-2.5} \mathrm{e}^{-1.191 f_{\mathrm{i}}-32.2 f_{\mathrm{m}}}{ }^{0.55} .
$$

Using $f_{\mathrm{i}}=f_{\mathrm{h}}^{0.735}=\left(1-\mathrm{e}^{-\left|P_{\mathrm{c}}-P\right| / P}\right)^{0.735}$, all of the parameters in Equations (3) and (5) can now be determined from $a b$ initio calculations. Equation (5) reveals that the metallic component $\left(f_{\mathrm{m}}\right)$ of a chemical bond has a stronger adverse effect on hardness than the ionic one $\left(f_{\mathrm{i}}\right)$. The $d$ valence electrons play an important role in increasing hardness by enhancing the directionality and consequentially the strength of the chemical bond. It should be noted that, for group IB and IIB metals with fully filled $d$ orbitals, the prefactor for the hardness calculation is 350 . The extension of this formula to multicomponent systems is similar to Equation (4), with attentions on the metallicity and prefactor for individual bonds [18].

Hardness predictions using Equations (3-5) have been extensively performed for a large class of theoretically designed superhard structures in recent years [52-72]. Our hardness model is applicable to polar covalent crystals, oxides with a partial contribution from ionic bonds, and ionic crystals, as well as some multicomponent crystals with mixed types of interatomic bonds [19]. The overall agreement with experiments is highly satisfactory. This consistency indicates that hardness can be defined micro- scopically as the combined resistance of chemical bonds in a crystal to indentation.

Following our seminal work, several microscopic hardness models have been proposed based on different treatments of the deformation resistance of the chemical bonds to the indenter, such as the bond strength model [20] and the electronegativity model [21]. All of these models are based on the assumption that hardness is equivalent to the sum of the resistance of each chemical bond to the indenter. The difference among these microscopic models is that the resistance is expressed differently in each: by the energy gap in our bond resistance model, by the bond strength consisting of a reference energy in the bond strength model, and by the bond electronegativity consisting of the element electronegativity in the electronegativity model. An overall analysis of these models sheds light on the factors that should be considered when designing superhard materials. These factors include short and strong chemical bonds, high valence electron density or high bond density, and strongly directional bonds (as suggested by the larger prefactor for $d$ orbitals). Ionicity is adverse for hardness, as clearly demonstrated by the exponential factor, and metallicity is even worse. Readers are encouraged to refer to our recent reviews for more detailed discussion and comparison of these models [19,73]. In Ref. [73], our recent progress in microscopic models of tensile strength and bulk modulus are also discussed. It has to be emphasized that, although microscopic hardness models have been established for (polar) covalent materials with some success, a satisfying, general description of hardness for covalent crystals, ionic crystals, and metals still eludes materials scientists because of the inherent complexities $[8,74,75]$.

\section{HARDER THAN DIAMOND}

Synthesis of superhard materials with hardness higher than that of diamond has always been a pursued goal. This is truly a challenge to materials science, and some researchers are not optimistic to fulfill this goal [76]. In general, there are two pathways for this ambition: one is to identify novel superhard single crystals; the other is to enhance the hardness of known materials through microstructure modulation. The systems considered in the first pathway belong to the intrinsic superhard materials where high hardness is achieved through their strong chemical bonding. However, a recent research indicates that design of novel single crystals harder than natural diamond seems unrealistic [77], leaving the second route as the only choice where extrinsic hardening can be achieved through nanostructuring. Recent progresses in $\mathrm{TiN} / \mathrm{SiN}_{x}$ nanocomposites [9], aggregated boron nitride nanocomposites [41], nanograined cBN (ng-cBN) [42] and diamond [40] evidenced the feasibility of the second pathway for hardness enhancement. 
Here we give some qualitative considerations of hardening effects due to nanostructuring in polycrystalline materials [19]. First, the dislocation activities inside the grain is suppressed with decreasing grain size due to the Hall-Petch effect, leading to the grain boundaries hardening described as $H=H_{0}+K_{\mathrm{HP}} / \sqrt{D}[78,79]$, where $H_{0}$ is the hardness of the bulk single crystal, $K_{\mathrm{HP}}$ is the Hall-Petch hardening coefficient, and $D$ is the grain size in nanometer. In addition, Tse et al. [80] suggested a quantum confinement hardening effect for nanocrystals. Based on the Kubo theory and considering the quantum confinement effect, the band gap $E_{\mathrm{g}}$ in Equation (1) should be updated as $E_{\mathrm{g} \text {,nano }}(\mathrm{eV})=E_{\mathrm{g}, \mathrm{bulk}}+\delta_{P}$ $=E_{\mathrm{g}, \mathrm{bulk}}+24 / D N_{\mathrm{e}}^{1 / 3}$ for nanocrystals. As a result, nanocrystal hardness is enhanced by $211 D^{-1} N_{\mathrm{e}}^{1 / 3} \mathrm{e}^{-1.191 f_{\mathrm{i}}}$ compared with that of the bulk single crystal. Combining the HallPetch and quantum confinement effects, the hardness for a nanocrystalline bulk material can be estimated as:

$$
H=H_{0}+K_{\mathrm{HP}} / \sqrt{D}+K_{\mathrm{qc}} / D,
$$

where $K_{\mathrm{qc}}=211 N_{\mathrm{e}}^{1 / 3} \mathrm{e}^{-1.191 \mathrm{f}_{\mathrm{i}}}$ is the quantum confinement hardening coefficient. This equation describes the observed relations between hardness and crystallite size in $\mathrm{cBN}$ and wurtzite BN (wBN) nanocomposites perfectly [41].

Suggested by Equation (6), a higher hardness would be expected with smaller grain size. In experiments, however, opposite variations of hardness were observed if the grain size is lower than $\sim 10 \mathrm{~nm}[41,81]$. The observed softening seems to originate from intergranular fracturing along poorly sintered grain boundaries rather than the reverse Hall-Petch effect resulting from grain-boundary sliding [81]. The synthesis of well-sintered nanograined superhard materials while maintaining a smaller grain size is technically difficult. It was known that coherent twin boundaries possess excess energy typically one order of magnitude lower than that of grain boundaries [82]. In addition, twin boundaries at nanoscale show a hardening effect identical to those of grain boundaries for metals $[82,83]$. Nanotwinning thus provides a more effective mechanism to achieve smaller characteristic size of microstructure. Accordingly, we managed to synthesize ultrahard $\mathrm{cBN}$ and diamond bulk materials with nanotwinned microstructures under high pressure and high temperature (HPHT).

Using onion-like BN nanoparticles $(30-150 \mathrm{~nm}$ in diameter) as precursor, we successfully synthesized transparent single-phase nt-cBN bulks under HPHT [43]. Typical microscopic structures of onion $\mathrm{BN}$ precursor and nt-cBN synthesized at $15 \mathrm{GPa}$ and $1800^{\circ} \mathrm{C}$ are demonstrated in Fig. 2 [43]. The naturally puckered BN layers, the high concentration of stacking faults, and the spherical shape of the precursor nanoparticles ensure the formation of laminated nanotwins in $\mathrm{cBN}$ nanograins (Fig. 2a). cBN nanograins contained densely spaced lamellar $\{111\}$ twins with an average twin thickness of $3.8 \mathrm{~nm}$ (Figs $2 \mathrm{~b}$ and c). Hardness and fracture toughness of the bulk nt-cBN samples were measured with a standard Vickers diamond indenter (Fig. 3). The formation of ultrathin nanotwins significantly improved the material's hardness and fracture toughness: a very high Vickers hardness (exceeding $100 \mathrm{GPa}$, the optimal hardness of synthetic diamond) and a large fracture toughness ( $>12 \mathrm{MPa} \mathrm{m} \mathrm{m}^{0.5}$, well beyond the toughness of commercial cemented tungsten carbide of $10 \mathrm{MPa} \mathrm{m}^{0.5}$ ) were achieved. An onset oxidation temperature of $1294^{\circ} \mathrm{C}$ was determined for nt-cBN, which is higher than those of single-crystal $\mathrm{cBN}\left(\sim 1103^{\circ} \mathrm{C}\right)$, ng-cBN $\left(\sim 1187^{\circ} \mathrm{C}\right)$ [42], and commercial polycrystalline $\mathrm{cBN}\left(\sim 1000^{\circ} \mathrm{C}\right)$ [84]. The simultaneous improvement of superhard tools' three key properties, namely hardness, toughness and stability, is accomplished, indicating the appealing application prospect of ultrahard $\mathrm{cBN}$ material.

Following the same principle, we successfully synthe-
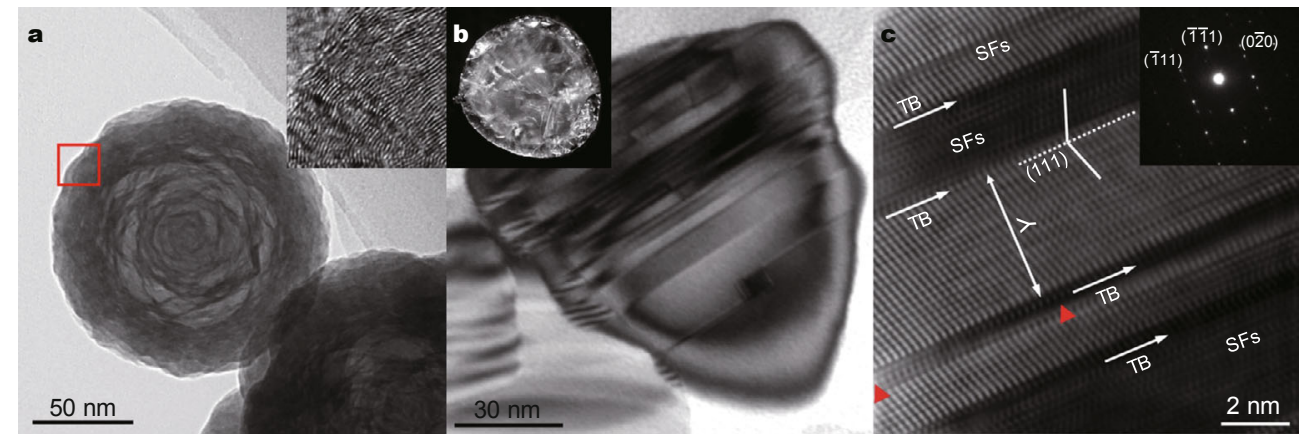

Figure 2 (a) Transmission electron microscopy (TEM) image of onion BN nanoparticles. The inset is high resolution TEM (HRTEM) image corresponding to the position marked with the red box emphasizing features of curved atomic layers, lattice puckering, and stacking faults. (b) Bright filed TEM image of a cBN nanograin. The inset shows an nt-cBN bulk sample with a diameter of $2 \mathrm{~mm}$. (c) HRTEM image and corresponding selected area electron diffraction pattern (SAED, inset) along the [101] zone axis of the nanograin shown in (b). Lamellar nanotwins with various thicknesses $(\lambda)$ are present in the nanograin. The twinning plane is of the $\{111\}$ type with lattice fringe angles of $70.53^{\circ}$ across the twin plane. The red triangles mark two Shockley dislocations. Reprinted with permission from Ref. [43]. Copyright 2013, Nature Publishing Group. 


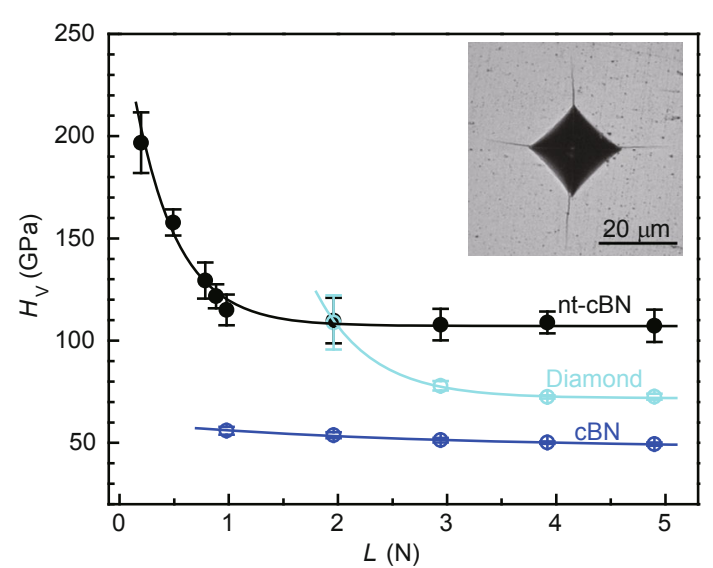

Figure 3 The Vickers hardness of an nt-cBN bulk sample, a synthetic diamond single crystal, and a cBN single crystal as functions of applied load. The inset shows an optical micrograph of the Vickers indentation at a load of $19.6 \mathrm{~N}$ with cracks produced for fracture toughness evaluation $\left(K_{\mathrm{IC}}=12.7 \mathrm{MPa} \mathrm{m}^{0.5}\right)$.

sized transparent nt-diamond bulks under HPHT by using onion carbon precursor with diameters of 20-50 nm [44]. Typical microscopic structures of onion carbon precursor and nt-diamond synthesized at $20 \mathrm{GPa}$ and $2000^{\circ} \mathrm{C}$ are demonstrated in Fig. 4 [44]. A high density of lamellar $\{111\}$ nanotwins was observed, which are predominantly thinner than $10 \mathrm{~nm}$ with an average twin thickness of $\sim 5$ nm. As shown in Fig. 5, this nanotwinned diamond shows excellent mechanical properties. Its Vickers hardness reaches unprecedented $200 \mathrm{GPa}$, twice that of natural diamond, and realizes the lasting dream of human beings (i.e., finding a material harder than natural diamond). In addition, the fracture toughness goes up to $15 \mathrm{MPa} \mathrm{m} \mathrm{m}^{0.5}$. The trade-off between hardness and toughness of our nt-diamond samples (Fig. 5b) is significantly superior to those of previously reported diamond-related materials [85-88].
These nt-diamond bulks also show an enhanced thermal stability with in-air oxidization temperature $200^{\circ} \mathrm{C}$ higher than that of natural diamond [44].

It is interesting to note that the old paradigm, i.e., the higher the hardness of a material, the lower the fracture toughness, is broken in both nt-cBN and nt-diamond bulks. The simultaneous improvement in hardness and fracture toughness in our nanotwinned samples is intimately related to the ubiquitous nanotwinning microstructure. The presence of ultrathin nanotwins introduces extra hardening, which can be attributed to the joint contribution from the Hall-Petch and quantum confinement effects at nanoscale, meanwhile gliding of dislocations along the densely distributed twin boundaries enhances fracture toughness [89]. Our findings demonstrate a new strategy to manufacture advanced superhard materials. By selecting suitable starting materials and optimal P-T conditions, interlocked nanotwinned microstructures can be created, and a simultaneous improvement of hardness, fracture toughness, and thermal stability of superhard materials is expected. Such advantages of nt-diamond and nt-cBN endow them wider applications in both industrial and scientific fields, such as turning, milling tools with better performance, and diamond anvil cell providing a pressure higher than $500 \mathrm{GPa}$.

\section{CRITERION OF INDENTATION HARDNESS MEASUREMENT}

Along with these progresses in superhard materials research, there exists a practical issue about hardness measurement: how can indentation hardness be reliably measured? Previously, it was proposed that "it is not yet possible to express the hardness of a material 'harder than diamond' by a single number. We recommend that values higher than 120 GPa should not be called 'hardness' to avoid confusion"

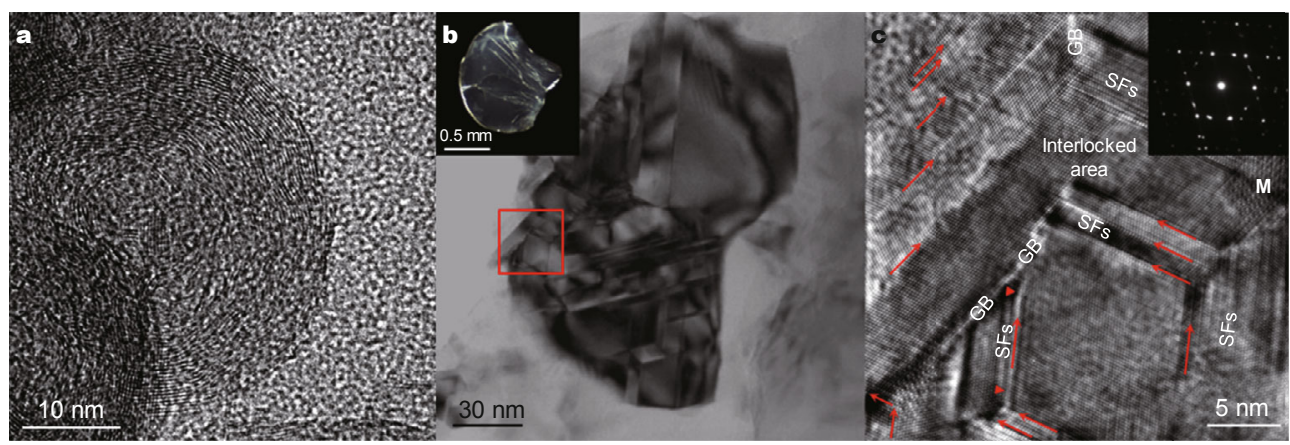

Figure 4 (a) HRTEM image of onion carbon nanoparticles. (b) TEM image of nanotwinned microstructure. The inset shows a photograph of the transparent sample ( $1 \mathrm{~mm}$ in diameter). (c) HRTEM image of intersecting nanotwins (marked with the red box in (b)), viewed along the [101] zone axis of diamond. Lamellar $\{111\}$ nanotwins, stacking faults are present. Twin boundaries are marked with red arrows. Grain boundaries (GB) are interrupted by interlocked twins. The inset shows SAED pattern corresponding to the central area of (b). The four-fold-like pattern is from the twin domains with four different orientations. Reprinted with permission from Ref. [44]. Copyright 2014, Nature Publishing Group. 

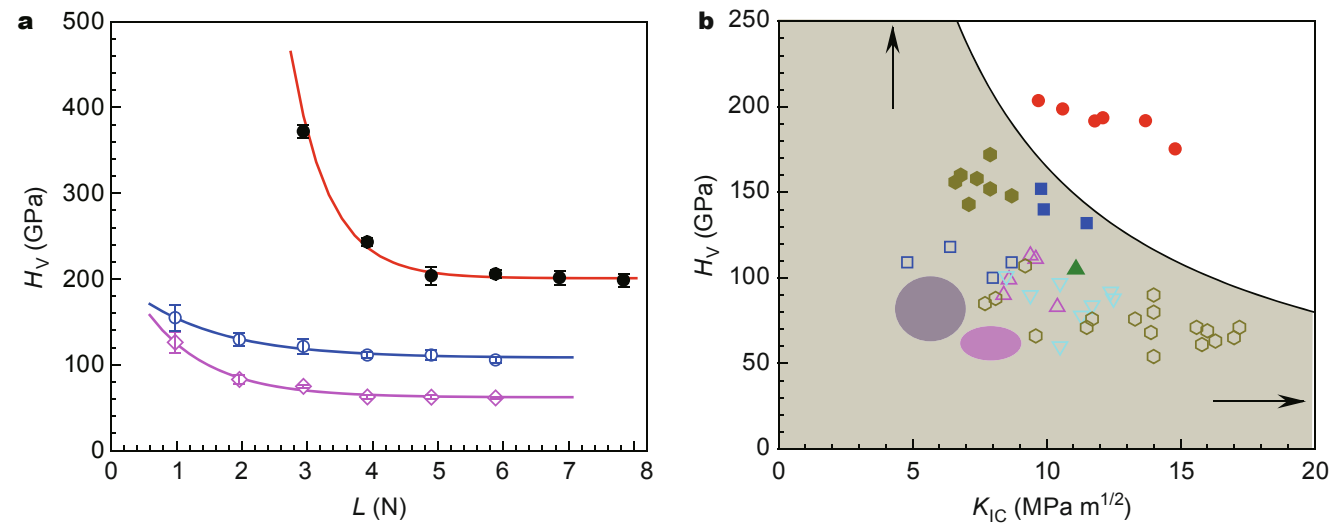

Figure 5 (a) The Vickers hardness of nt-diamond and natural diamond crystal as a function of applied load. Beyond $4.9 \mathrm{~N}, H_{\mathrm{V}}$ decreases to the asymptotic values of $\sim 200 \mathrm{GPa}$ for nt-diamond (red line). For natural diamond crystals, our measured $H_{\mathrm{V}}$ values are $\sim 110 \mathrm{GPa}$ on the (110) face (blue line) and $\sim 62 \mathrm{GPa}$ on the (111) face (pink line). (b) Plot of $H_{\mathrm{V}}$ against $K_{\mathrm{IC}}$ for nt-diamond (solid red circle) in comparison with available data on other forms of diamond. Open upward triangle: type Ia natural diamond single crystal (SC-D), open square: type IIa natural SC-D, open downward triangle: HPHTgrown SC-D, open hexagon: CVD grown SC-D, filled squares: annealed IIa natural SC-D, and filled hexagons: CVD-grown and HPHT annealed SC-D [85]; large grey circle: Co-polycrystalline diamond (PCD) [86]; large pink oval: CVD-grown PCD [87]; filled upward triangle: aggregated diamond rod [88]. Reprinted with permission from Ref. [44]. Copyright 2014, Nature Publishing Group.

[90]. This traditional criterion (hereinafter referred to as the comparison criterion) implies that the test sample must be softer than the natural diamond indenter (with hardness of $120 \mathrm{GPa}$ ) to ensure a hardness measurement. If the comparison criterion were correct, it would be impossible to characterize the hardness for these materials. However, experimentally there exist many counterexamples to the comparison criterion. For example, Vickers hardness of the annealed chemical vapor deposition (CVD) diamond as high as $170 \mathrm{GPa}$ was reported previously [85], significantly higher than the suggested limit of $120 \mathrm{GPa}$. A scratch made by $\mathrm{ReB}_{2}$ on the surface of diamond was verified by atomic force microscopy even through $\mathrm{ReB}_{2}$ is much softer than diamond [91]. Another example contradict to the comparison criterion is the soft impressor method developed by Brookes et al. [92], where a plastic deformation can be formed even though the hardness of the impressor is much lower than that of the tested sample $[93,94]$. The successful application of the soft impressor method is that the resolved shear stress exceeds those requirements for dislocation initiation and multiplication [94]. To clarify this controversy, we now recall the definition of indentation hardness. The indentation hardness of a material is determined by the indenter load divided by the contact (or projected) area of the permanent indentation formed on the sample surface [11]. According to this definition, indentation hardness is a well-defined "engineering" quantity and can be measured as long as a permanent indentation (through plastic deformation) can be left on the surface of the tested sample. Obviously, the question about the criterion to perform an indentation hardness measurement comes down to the condition of forming a permanent indentation on the sur- face. The specific stress states of the indenter and tested sample during an indentation hardness measurement are analyzed to identify this condition $[95,96]$.

When the symmetric indentor is exactly perpendicular pushed into the tested sample, the horizontal force components from opposite facets of the indenter are offset, leaving only the perpendicular compressive force components to apply on the indenter. The tip of the diamond indenter is thus subjected to a compressive stress field. The stress state of the sample is different from that of the indenter though. In the sample tested zone surrounding the indenter, dislocation initiation and multiplication cause slips and plastic strain when the applied stress exceeds the shear strength of the sample, leading to a plastic shear deformation of the sample and the formation of a permanent indentation on the sample surface. Indentation hardness can thus be measured reliably as long as the shear strength of the sample is smaller than the compressive strength of the indenter diamond $[95,96]$. The compressive strengths, $\sigma$, of diamond are $223 \mathrm{GPa}$ in the weakest $\langle 100\rangle$ direction, and about 470 GPa along $\langle 110\rangle$ and $\langle 111\rangle$ [97]. The shear strengths, $\tau$, of hard and superhard materials are significantly lower than the compressive strengths of diamond [38,98-102], ensuring the formation of an indentation. For example, the shear strength of superhard diamond, $\mathrm{cBN}, \mathrm{wBN}$, and $\mathrm{B}_{6} \mathrm{O}$ are 93 [98], 58 [99], 62 [99] and $38 \mathrm{GPa}$ [102], respectively. Therefore, hardness measured exceeding that of natural diamond indenter does have physical meaning and is reliable.

\section{PROSPECTS}

Inspired by the success in nt-cBN and nt-diamond, we expect more achievements in superhard materials through 
the strategy of nanostructuring. In the following, several topics of special interest are discussed. First, pursuing microstructure with thinner nanotwin sizes may lead to superhard materials with even superior properties. Here we estimate the lower limit of nanotwin thickness and the corresponding ultimately achievable hardness (UAH) of diamonds [44]. Taking $\{111\}$ twins in nt-diamond as the model system, the minimal twin thickness is $\lambda_{\min }=3 \times$ $d_{111}=0.618 \mathrm{~nm}$ since the atomic stacking sequence along the $\langle 111\rangle$ direction of diamond is $\cdots$ ABCABC $\cdots$. At such length scale, the Hall-Petch effect is no longer applicable $[103,104]$. With $H_{0}=90 \mathrm{GPa}$ (hardness of single-crystal diamond) and $K_{\mathrm{qc}}=211 N_{\mathrm{e}}^{1 / 3}=187.7 \mathrm{GPa} \mathrm{nm}$, an extraordinary UAH of $394 \mathrm{GPa}$ is estimated for nt-diamond from Equation (6) (without contribution of the Hall-Petch effect). It calls for a technical challenge to synthesize nanotwinned microstructures with the required twin thickness to achieve such an exceptional hardness property. If we can refine the precursor (onion carbon and onion $\mathrm{BN}$ ) with smaller nanoparticle size, further decrease of twin thickness in the synthetic nanotwinned bulks and consequential increase in hardness are possible. In addition, precursors other than the onion-liked ones will be investigated under HPHT, such as the self-oriented carbon nanotube arrays and yarns $[105,106]$. The formation mechanism of the nanotwins may be revealed by comparison of HPHT products from spherical carbon onions and tubular carbon nanotubes. Note that the experimental HPHT conditions for synthesizing nt-diamond and nt-cBN are essentially identical. This opens up the possibility of manufacturing nt-diamond/nt-cBN nanocomposites. Such nanotwinned composites are expected to possess intermediate oxidation temperature and hardness between those of nt-diamond and nt-cBN but with greater fracture toughness as a result of the combined contributions from nanotwinning and composite effects.

With nanostructuring, it is possible to tune a variety of hard materials $\left(H_{\mathrm{V}}>15 \mathrm{GPa}\right)$ into superhard materials, and enrich the family of superhard materials. The key is, along with the decrease of nanograins size, the strong grain boundaries must be maintained to avoid the intergranular fracturing that induces hardness deficiency. Most recently, it was demonstrated that the Hall-Petch effect still functions for fully dense spinel $\mathrm{MgAl}_{2} \mathrm{O}_{4}$ nanocrystalline ceramics [107]. Sintering $\mathrm{MgAl}_{2} \mathrm{O}_{4}$ nanopowders with an average size of $25.8 \mathrm{~nm}$ under $2 \mathrm{GPa}$ and $740^{\circ} \mathrm{C}$, nanocrystalline bulks with an average grain size of $28 \mathrm{~nm}$ were achieved, leading to a unique hardness of $20.2 \mathrm{GP}\left(H_{0}=13 \mathrm{GPa}\right.$ for $\mathrm{MgAl}_{2} \mathrm{O}_{4}$ single crystal). With carefully prepared precursor, smaller nanograin size, and higher pressure to restrict the grain growth, well-sintered nanocrystalline hard materials may be promoted into the family of superhard materials.
Received 10 December 2014; accepted 8 January 2015

published online 10 February 2015

1 Haines J, Leger JM, Bocquillon G. Synthesis and design of superhard materials. Annu Rev Mater Res, 2001, 31: 1-23

2 Man-made diamonds. Chem Eng News, 1955, 33: 718

3 Wentorf RH. Cubic form of boron nitride. J Chem Phys, 1957, 26: 956-956

4 Gilman JJ, Cumberland RW, Kaner RB. Design of hard crystals. Int J Refract Met Hard Mater, 2006, 24: 1-5

5 Ivanovskii AL. The search for novel superhard and incompressible materials on the basis of higher borides of $s, p, d$ metals. J Superhard Mater, 2011, 33: 73-87

6 Riedel R. Novel ultrahard materials. Adv Mater, 1994, 6: 549-560

7 Veprek S. Recent search for new superhard materials: go nano! J Vac Sci Technol A, 2013, 31: 050822

8 Kaner RB, Gilman JJ, Tolbert SH. Designing superhard materials. Science, 2005, 308: 1268-1269

9 Veprek S. The search for novel, superhard materials. J Vac Sci Technol A, 1999, 17: 2401-2420

10 Brazhkin VV, Lyapin AG, Hemley RJ. et al. Harder than diamond: dreams and reality. Philos Mag A, 2002, 82: 231-253

11 Meyer E. Analysis of hardness examination and hardness. Zt Vereins Deutscher Ingenieure, 1908, 52: 645-654

12 Gilman JJ. The Science of Hardness Testing and Its Research Applications. Metals Park: American Society for Metals, 1973

13 Liu AY, Cohen ML. Prediction of new low compressibility solids. Science, 1989, 245: 841-842

14 Teter DM. Computational alchemy: the search for new superhard materials. MRS Bull, 1998, 23: 22-27

15 Chen XQ, Niu H, Li D, et al. Modeling hardness of polycrystalline materials and bulk metallic glasses. Intermetallics, 2011, 19: 12751281

16 Gao FM, He JL, Wu ED, et al. Hardness of covalent crystals. Phys Rev Lett, 2003, 91: 015502

$17 \mathrm{He}$ JL, Wu ED, Wang HT, et al. Ionicities of boron-boron bonds in $\mathrm{B}_{12}$ icosahedra. Phys Rev Lett, 2005, 94: 015504

18 Guo X, Li L, Liu Z, et al. Hardness of covalent compounds: roles of metallic component and d valence electrons. J Appl Phys, 2008, 104: 023503

19 Tian Y, Xu B, Zhao Z, et al. Microscopic theory of hardness and design of novel superhard crystals. Int J Refract Met Hard Mater, 2012, 33: 93-106

20 Simunek A, Vackar J. Hardness of covalent and ionic crystals: first-principle calculations. Phys Rev Lett, 2006, 96: 085501

21 Li KY, Wang XT, Zhang FF, et al. Electronegativity identification of novel superhard materials. Phys Rev Lett, 2008, 100: 235504

22 Zhao Y, He DW, Daemen LL, et al. Superhard B-C-N materials synthesized in nanostructured bulks. J Mater Res, 2002, 17: 3139-3145

23 Solozhenko VL, Gregoryanz E. Synthesis of superhard materials. Mater Today, 2005, 8: 44-51

24 Solozhenko VL, Andrault D, Fiquet G, et al. Synthesis of superhard cubic $\mathrm{BC}_{2} \mathrm{~N}$. Appl Phys Lett, 2001, 78: 1385-1387

25 Solozhenko VL, Kurakevych OO, Andrault D, et al. Ultimate metastable solubility of boron in diamond: synthesis of superhard diamondlike BC $_{5}$. Phys Rev Lett, 2009, 102: 015506

26 Solozhenko VL, Kurakevych OO, Oganov AR, et al. On the hardness of a new boron phase, orthorhombic $\gamma-B_{28}$. J Superhard Mater, 2008, 30: 428-429

27 He DW, Zhao YS, Daemen L, et al. Boron suboxide: as hard as cubic boron nitride. Appl Phys Lett, 2002, 81: 643-645

28 Levine JB, Tolbert SH, Kaner RB, et al. Advancements in the search for superhard ultra-incompressible metal borides. Adv Funct Mater, 2009, 19: 3519-3533

29 Gu Q, Krauss G, Steurer W, et al. Transition metal borides: super- 
hard versus ultra-incompressihle. Adv Mater, 2008, 20: 3620-3626

30 Chung HY, Weinberger MB, Levine JB, et al. Synthesis of ultra-incompressible superhard rhenium diboride at ambient pressure. Science, 2007, 316: 436-439

31 Crowhurst JC, Goncharov AF, Sadigh B, et al. Synthesis and characterization of the nitrides of platinum and iridium. Science, 2006, 311: 1275-1278

32 Cumberland RW, Weinberger MB, Gilman JJ, et al. Osmium diboride, an ultra-incompressible, hard material. J Am Chem Soc, 2005, 127: 7264-7265

33 Ono S, Kikegawa T, Ohishi Y, et al. A high-pressure and high-temperature synthesis of platinum carbide. Solid State Commun, 2005, 133: 55-59

34 Qin J, He D, Wang J, et al. Is rhenium diboride a superhard material? Adv Mater, 2008, 20: 4780-4783

35 Young AF, Sanloup C, Gregoryanz E, et al. Synthesis of novel transition metal nitrides $\mathrm{IrN}_{2}$ and $\mathrm{OsN}_{2}$. Phys Rev Lett, 2006, 96: 155501

36 Gou H, Dubrovinskaia N, Bykova E, et al. Discovery of a superhard iron tetraboride superconductor. Phys Rev Lett, 2013, 111: 157002

37 Dubrovinskaia N, Dubrovinsky L, Solozhenko VL, et al. Comment on "Synthesis of ultra-incompressible superhard rhenium diboride at ambient pressure". Science, 2007, 318: 1550-1550

38 Li B, Sun H, Chen C, et al. First-principles calculation of the indentation strength of $\mathrm{FeB}_{4}$. Phys Rev B, 2014, 90: 014106

39 Zhang M, Lu M, Du Y, et al. Hardness of $\mathrm{FeB}_{4}$ : density functional theory investigation. J Chem Phys, 2014, 140: 174505

40 Irifune T, Kurio A, Sakamoto S, et al. Ultrahard polycrystalline diamond from graphite. Nature, 2003, 421: 599-600

41 Dubrovinskaia N, Solozhenko VL, Miyajima N, et al. Superhard nanocomposite of dense polymorphs of boron nitride: noncarbon material has reached diamond hardness. Appl Phys Lett, 2007, 90: 101912

42 Solozhenko VL, Kurakevych OO, Le Godec Y, et al. Creation of nanostuctures by extreme conditions: high-pressure synthesis of ultrahard nanocrystalline cubic boron nitride. Adv Mater, 2012, 24: 1540-1544

43 Tian Y, Xu B, Yu D, et al. Ultrahard nanotwinned cubic boron nitride. Nature, 2013, 493: 385-388

44 Huang Q, Yu D, Xu B, et al. Nanotwinned diamond with unprecedented hardness and stability. Nature, 2014, 510: 250-253

45 Pugh SF. Relations between the elastic moduli and the plastic properties of polycrystalline pure metals. Philos Mag, 1954, 45: 823-843

46 Chen XQ, Niu H, Franchini C, et al. Hardness of T-carbon: density functional theory calculations. Phys Rev B, 2011, 84: 121405

47 Nix WD, Gao H. Indentation size effects in crystalline materials: a law for strain gradient plasticity. J Mech Phys Solids, 1998, 46: 411-425

48 Gilman JJ. Flow of covalent solids at low-temperatures. J Appl Phys, 1975, 46: 5110-5113

49 Phillips JC. Ionicity of chemical bond in crystals. Rev Mod Phys, 1970, 42: 317-356

50 Siethoff $\mathrm{H}$. Homopolar band gap and thermal activation parameters of plasticity of diamond and zinc-blende semiconductors. J Appl Phys, 2000, 87: 3301-3305

51 Pauling L. The Nature of the Chemical Bond and the Structure of Molecules and Crystals: an Introduction to Modern Structural Chemistry. New York: Cornell University Press, 1960

52 Liu HY, Li QA, Zhu L, et al. Superhard polymorphs of diamond-like BC $_{7}$. Solid State Commun, 2011, 151: 716-719

53 Liu HY, Li QA, Zhu L, et al. Superhard and superconductive polymorphs of diamond-like $\mathrm{BC}_{3}$. Phys Lett A, 2011, 375: 771-774

54 Li Q, Chen WJ, Xia Y, et al. Superhard phases of $\mathrm{B}_{2} \mathrm{O}$ : an isoelectronic compound of diamond. Diamond Relat Mater, 2011, 20: 501-504

55 Li QA, Wang H, Tian YJ, et al. Superhard and superconducting structures of $\mathrm{BC}_{5}$. J Appl Phys, 2010, 108: 023507
56 Fan XF, Wu HY, Shen ZX, et al. A first-principle study on the structure, stability and hardness of cubic $\mathrm{BC}_{2} \mathrm{~N}$. Diamond Relat Mater, 2009, 18: 1278-1282

57 Guo X, Liu Z, Luo X, et al. Theoretical hardness of the cubic $\mathrm{BC}_{2} \mathrm{~N}$. Diamond Relat Mater, 2007, 16: 526-530

$58 \mathrm{Li} \mathrm{Y,} \mathrm{Li} \mathrm{Q,} \mathrm{Ma} \mathrm{Y,} \mathrm{et} \mathrm{al.} \mathrm{B}_{2} \mathrm{CO}$ : a potential superhard material in the B-C-O system. Europhys Lett, 2011, 95: 66006

59 Li DX, Yu DL, He JL, et al. First-principle calculation on structures and properties of diamond-like $\mathrm{B}_{3} \mathrm{C}_{10} \mathrm{~N}_{3}$ compound. J Alloys Compd, 2009, 481: 855-857

60 Zhao Z, Cui L, Wang LM, et al. Bulk $\mathrm{Re}_{2} \mathrm{C}$ : crystal structure, hardness, and ultra-incompressibility. Cryst Growth Des, 2010, 10: 5024-5026

61 Wang ZH, Kuang XY, Zhong MM, et al. Pressure-induced structural transition of $\mathrm{OsN}_{2}$ and effect of metallic bonding on its hardness. Europhys Lett, 2011, 95: 66005

62 Shao X. Prediction of a low-dense $\mathrm{BC}_{2} \mathrm{~N}$ phase. Chin Phys Lett, 2011, 28: 057101

63 Wang $\mathrm{H}$, Li Q, Wang $\mathrm{H}$, et al. Design of superhard ternary compounds under high pressure: $\mathrm{SiC}_{2} \mathrm{~N}_{4}$ and $\mathrm{Si}_{2} \mathrm{CN}_{4}$. J Phys Chem C, 2010, 114: 8609-8613

64 Hu M, Zhao Z, Tian F, et al. Compressed carbon nanotubes: a family of new multifunctional carbon allotropes. Sci Rep, 2013, 3: 1331

65 Zhao Z, Tian F, Dong X, et al. Tetragonal allotrope of group 14 elements. J Am Chem Soc, 2012, 134: 12362-12365

66 Zhao Z, Xu B, Zhou XF, et al. Novel superhard carbon: C-centered orthorhombic $C_{8}$. Phys Rev Lett, 2011, 107: 215502

67 Xiong M, Fan CZ, Zhao ZS, et al. Novel three-dimensional boron nitride allotropes from compressed nanotube bundles. J Mater Chem C, 2014, 2: 7022-7028

68 Amsler M, Flores-Livas J, Lehtovaara L, et al. Crystal structure of cold compressed graphite. Phys Rev Lett, 2012, 108: 065501

69 Baburin IA, Proserpio DM, Saleev VA, et al. From zeolite nets to $\mathrm{sp}^{3}$ carbon allotropes: a topology-based multiscale theoretical study. Phys Chem Chem Phys, 2015, 17: 1332-1338

$70 \mathrm{Bu} \mathrm{H}$, Zhao M, Dong W, et al. A metallic carbon allotrope with superhardness: a first-principles prediction. J Mater Chem C, 2014, 2: $2751-2757$

71 Zhang M, Liu H, Du Y, et al. Orthorhombic C32: a novel superhard $\mathrm{sp}^{3}$ carbon allotrope. Phys Chem Chem Phys, 2013, 15: 1412014125

72 Hu M, Huang Q, Zhao Z, et al. Superhard and high-strength ynediamond semimetals. Diamond Relat Mater, 2014, 46: 15-20

73 Xu B, Tian YJ. Microscopic models of materials mechanical properties. Prog Phys, 2014, 34: 191-201

74 Gilman JJ. Chemistry and Physics of Mechanical Hardness. Hoboken: John Wiley \& Sons, 2009

75 Tse JS. Intrinsic hardness of crystalline solids. J Superhard Mater, 2010, 32: 177-191

76 Chaudhri MM, Lim YY. Harder than diamond? Just fiction. Nat Mater, 2005, 4: 4

77 Zhang X, Wang Y, Lv J, et al. First-principles structural design of superhard materials. J Chem Phys, 2013, 138: 114101

78 Hall EO. The deformation and ageing of mild steel: III. discussion of results. Proc Phys Soc London B, 1951, 64: 747-753

79 Petch NJ. The cleavage strength of polycrystals. J Iron Steel Ins, 1953, 174: 25-28

80 Tse JS, Klug DD, Gao F. Hardness of nanocrystalline diamonds. Phys Rev B, 2006, 73: 140102

81 Sumiya H, Irifune T. Hardness and deformation microstructures of nano-polycrystalline diamonds synthesized from various carbons under high pressure and high temperature. J Mater Res, 2007, 22: 2345-2351

82 Lu L, Chen X, Huang X, et al. Revealing the maximum strength in nanotwinned copper. Science, 2009, 323: 607-610 
83 Lu L, Shen Y, Chen X, et al. Ultrahigh strength and high electrical conductivity in copper. Science, 2004, 304: 422-426

84 Sumiya H, Uesaka S, Satoh S, et al. Mechanical properties of high purity polycrystalline $\mathrm{cBN}$ synthesized by direct conversion sintering method. J Mater Sci, 2000, 35: 1181-1186

85 Yan CS, Mao HK, Li W, et al. Ultrahard diamond single crystals from chemical vapor deposition. Phys Status Solidi A, 2004, 201: R25-R27

86 Lammer A. Mechanical properties of polycrystalline diamonds. Mater Sci Technol, 1988, 4: 949-955

87 Sussmann RS, Pickles CSJ, Brandon JR, et al. CVD diamond windows for infrared synchrotron applications. Il Nuovo Cimento D, 1998, 20: 503-525

88 Dubrovinskaia N, Dub S, Dubrovinsky L, et al. Superior wear resistance of aggregated diamond nanorods. Nano Lett, 2006, 6: 824-826

$89 \mathrm{Lu} \mathrm{K}, \mathrm{Lu} \mathrm{L}$, Suresh S, et al. Strengthening materials by engineering coherent internal boundaries at the nanoscale. Science, 2009, 324: 349-352

90 Brazhkin V, Dubrovinskaia N, Nicol M, et al. What does "harder than diamond" mean? Nat Mater, 2004, 3: 576-577

91 Chung HY, Weinberger MB, Levine JB, et al. Response to comment on "Synthesis of ultra-incompressible superhard rhenium diboride at ambient pressure". Science, 2007, 318: 1550

92 Brookes CA, Green P. Deformation of magnesium-oxide crystals by softer indenters and sliders. Nature Phys Sci, 1973, 246: 119-122

93 Brookes CA, Shaw MP. Cumulative deformation of magnesium-oxide crystals by softer sliders. Nature, 1976, 263: 760-762

94 Brookes EJ, Comins JD, Daniel RD, et al. A study of plastic deformation profiles of impressions in diamond. Diamond Relat Mater, 2000, 9: 1115-1119

95 Tian Y, Xu B, Yu D, et al. Controversy about ultrahard nanotwinned cBN . Nature, 2013, 502: E1-E2

$96 \mathrm{Xu}$ B, Tian YJ. Ultrahardness: measurement and enhancement. J Phys Chem C, In press

97 Luo X, Liu Z, Xu B, et al. Compressive strength of diamond from first-principles calculation. J Phys Chem C, 2010, 114: 17851-17853

98 Roundy D, Cohen M. Ideal strength of diamond, Si, and Ge. Phys
Rev B, 2001, 64: 212103

99 Zhang R, Veprek S, Argon A, et al. Anisotropic ideal strengths and chemical bonding of wurtzite $\mathrm{BN}$ in comparison to zincblende $\mathrm{BN}$. Phys Rev B, 2008, 77: 172103

100 Zhang RF, Sheng SH, Veprek S, et al. First principles studies of ideal strength and bonding nature of AlN polymorphs in comparison to TiN. Appl Phys Lett, 2007, 91: 031906

101 Umeno Y, Shiihara Y, Yoshikawa N, et al. Ideal shear strength under compression and tension in $\mathrm{C}, \mathrm{Si}, \mathrm{Ge}$, and cubic $\mathrm{SiC}$ : an ab initio density functional theory study. J Phys Condens Matter, 2011, 23: 385401

102 Zhang RF, Legut D, Wen XD, et al. Bond deformation paths and electronic instabilities of ultraincompressible transition metal diborides: case study of $\mathrm{OsB}_{2}$ and $\mathrm{IrB}_{2}$. Phys Rev B, 2014, 90: 094115

103 Yip S. Nanocrystals: the strongest size. Nature, 1998, 391: 532-533

104 Li X, Wei Y, Lu L, et al. Dislocation nucleation governed softening and maximum strength in nano-twinned metals. Nature, 2010, 464: $877-880$

105 Jiang K, Li Q, Fan S, et al. Nanotechnology: spinning continuous carbon nanotube yarns. Nature, 2002, 419: 801

106 Fan S, Chapline MG, Franklin NR, et al. Self-oriented regular arrays of carbon nanotubes and their field emission properties. Science, 1999, 283: 512-514

107 Wollmershauser JA, Feigelson BN, Gorzkowski EP, et al. An extended hardness limit in bulk nanoceramics. Acta Mater, 2014, 69: 9-16

Acknowledgments This work was supported by the National Natural Science Foundation of China (51421091 and 51332005), and the Natural Science Foundation for Distinguished Young Scholars of Hebei Province of China (E2014203150).

Author contributions Tian Y initiated and guided the whole project. Xu $\mathrm{B}$ and Tian Y investigated the literature, discussed the content, and wrote the manuscript.

Conflict of interest The authors declare that they have no conflict of interest. 




Bo Xu is a professor of the College of Materials Science and Engineering, Yanshan University. He received his PhD from University of Nebraska-Lincoln (2002). After postdoctoral work at University of Maryland, College Park, he joined the State Key Laboratory of Metastable Materials Science and Technology, Yanshan University in 2006. His research interests include superhard materials and thermoelectric materials.

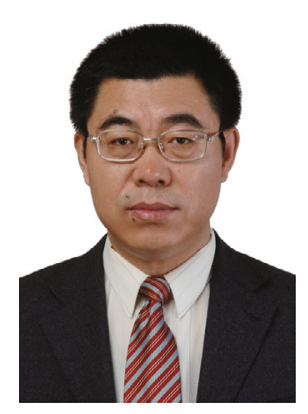

Yongjun Tian is a professor of the College of Materials Science and Engineering, Yanshan University. He received his PhD from Institute of Physics, Chinese Academy of Sciences, in 1994, and worked as a postdoctoral fellow in Universität Jena supported by the Humboldt Research Fellowships from 1996 to 1998. His research interests include design and synthesis of novel metastable materials.

中文摘要 半个世纪以来, 超硬材料研究领域一直存在两个重要难题亟待解决: (1) 硬度的微观理论或超硬材料的设计原理; (2) 合成出 综合性能(硬度、韧性和热稳定性)更加优异的新型超硬材料, 尤其是实现人工材料比天然金刚石更硬这一梦想. 在过去的十多年里, 我 们提出了一个基本假设一微观上硬度等于晶体单位面积中化学键对压入的综合阻抗, 由此建立了共价和极性共价晶体硬度的微观模 型, 实现了晶体硬度的定量预测. 随后, 我们将晶体硬度的微观模型推广至多晶固体, 建立了多晶共价材料硬度的理论模型. 最近, 以多 晶硬度模型为指导, 我们在高温高压条件下分别合成出纳米孪晶结构的立方氮化嗍和金刚石块材. 与单晶材料相比, 纳米孪晶立方氮 化硼和金刚石的硬度、韧性和热稳定性均得到明显改善, 为发展高性能超硬材料探索出一条崭新的途径. 